Résumés des conférences et travaux

\title{
Philologie des textes bouddhiques d'Asie Centrale
}

\section{Georges-Jean Pinault}

\section{OpenEdition \\ Journals}

Édition électronique

URL : https://journals.openedition.org/ashp/1072

DOI : 10.4000/ashp.1072

ISSN : 1969-6310

Éditeur

Publications de l'École Pratique des Hautes Études

\section{Édition imprimée}

Date de publication : 2 février 2011

Pagination : 369-372

ISSN : 0766-0677

\section{Référence électronique}

Georges-Jean Pinault, «Philologie des textes bouddhiques d'Asie Centrale », Annuaire de l'École pratique des hautes études (EPHE), Section des sciences historiques et philologiques [En ligne], 141 | 2011, mis en ligne le 25 février 2011, consulté le 06 juillet 2021. URL : http://journals.openedition.org/ashp/ 1072 ; DOl : https://doi.org/10.4000/ashp.1072 


\title{
PHILOLOGIE DES TEXTES BOUDDHIQUES D'ASIE CENTR ALE
}

\author{
Directeur d'études : M. Georges-Jean Pinault
}

Programme de l'année 2008-2009 : I. Linguistique indo-iranienne : composition nominale. II. Lecture de textes tokhariens.

\section{Linguistique indo-iranienne : composition nominale}

L'analyse de divers types de composés indo-iraniens s'est poursuivie par l'étude d'un problème qui a des conséquences importantes pour la reconstruction indoeuropéenne : celui des composés (et pseudo-composés) qui comportent en second membre véd. páti- «maître, chef ». Comme on sait, ces composés déterminatifs ont pris pour modèle le syntagme univerbé du type indo-eur. *dém-s póti- « maître de maison » > véd. dám-pati-, cf. véd. pátir dán (avec dán < *dán-s < indo-eur. *dém-s),

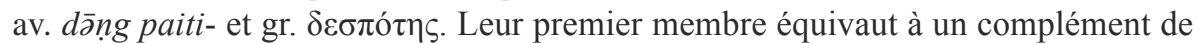
nom au génitif. La langue des hymnes védiques comporte en fait trois types de constructions avec páti-comme second terme : 1) des pseudo-composés avec pátiaccentué et une forme de génitif (authentique ou analogique) en premier membre, e. g. bráhmanas-páti-, vánas-páti-, ráthas-páti-;2) des composés (assez rares) avec páti- accentué et un thème inaccentué en premier membre, type rayi-páti- « maître de(s) richesse(s) », viś-páti- «maitre du clan »; 3) des composés, les plus nombreux, qui suivent le modèle de dám-pati-, et qui sont accentués sur le premier membre; celui-ci présente son accent propre, en sorte que la remontée d'accent propre au vocatif en début d'énoncé ne peut pas rendre compte du phénomène. Il faut signaler que, plus tard en védique, apparaissent des composés déterminatifs avec second membre -patí(au lieu de -páti-) : cette accentuation est due à l'influence des composés à second membre verbal régissant, qui sont oxytons, et des noms d'agent du type vavrí- «qui couvre », dadí- « qui donne », qui sont accentués sur la dernière syllabe. Jusqu'à présent, aucune théorie n'a rendu compte de l'accentuation aberrante des composés du type grhá-pati-, gó-pati-, vásu-pati-, etc., sauf l'idée spéculative qui en ferait originellement des composés possessifs (bahuvrīhi), glosés par « qui a la maîtrise de $\mathrm{N}$ » (N représenté par le premier membre), ce qui supposerait que páti- < indo-eur. *pót-i-

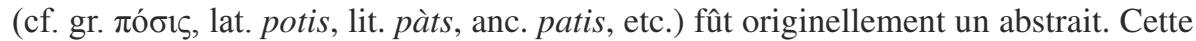
théorie est totalement contradictoire avec l'antiquité évidente du type dám-pati- et reste en l'air tant qu'il n'existe pas de racine verbale *pet- « commander, dominer », dont cet abstrait (« domination», vel sim.) serait le dérivé primaire. Or, il s'avère plutôt que *pot- est un élément para-nominal, associé à la particule intensive et exclusive reflétée par hitt. -pat, lit. pàt, lat. -pte, etc. Une analyse exhaustive des données védiques a permis de trouver la règle synchronique de distribution accentuelle entre -páti- et -pati- : sont accentués sur le second membre comme on l'attend pour des tatpurușa (composés déterminatifs) ceux qui coexistent avec un juxtaposé construit 
avec le génitif singulier en -as, type viś-páti- en regard de viśás (...) páti-. Cela exclut évidemment le type dám-pati-, qui constitue un archaïsme, puisque le génitif sg. correspondant ne se termine pas par -as. Par conséquent, les composés du type vásu-patidoivent s'expliquer par un autre type de syntagme, construit de préférence avec le génitif pluriel : il s'agirait du syntagme intensif, superlatif, vásūnām vásu- « bon parmi les bons », dont une forme renforcée, et équivalente pour la valeur intensive, était vásūnām vásu-pati-. Cette observation prolonge les remarques de C. Watkins (How to kill a dragon. Aspects of Indo-European poetics, New York - Oxford, 1995, p. 241245), qui se limitent à la dimension stylistique. Elle a évidemment des conséquences pour l'interprétation du nom indo-eur. *pót-i- «maître, chef ». On a posé que le sens de «maître » n'est pas issu simplement de la valeur primitive d'ipséité (comme le voulait E. Benveniste, voir Problèmes de linguistique générale, t. I, Paris, 1966, p. 301306; Le vocabulaire des institutions indo-européennes, Paris, 1969, t. I, p. 89-91, parce que le maître incarnerait l'unité sociale dont il est le chef). La notion de maîtrise, de puissance, ne peut se réduire à l'origine pronominale; elle est due à l'existence de ces syntagmes intensifs avec complément au génitif, où le dernier terme acquérait une valeur d'excellence et de supériorité. D'une certaine façon, Benveniste a esquissé la solution en supposant que le sens de « maitre » proviendrait des composés (op. cit., p. 305). Cela conduit évidemment à revoir complètement le dossier de la formation de

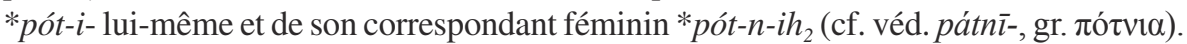
Du fait de ce dernier nom, qui a la même structure que $* h_{3} r \bar{e} \hat{g}-n-i h_{2}$ "reine » (cf. véd. rấjīì-, v.irl. rigain, lat. rēgìna <* rēgnīna), l'analyse ultime est étroitement liée à celle du type *déiw-ih « déesse » (véd. devít-), thème faible *diw-yéh ${ }^{-}$(gr. $\left.\delta \tilde{\imath} \alpha\right)$, qui constitue le modèle de la flexion de « maîtresse » et de « reine ». Hypothèse : le sens originel de *déiw-ih $h_{2}$ était « maîtresse du ciel », et cette notion de maîtrise, de domination provient de son « patron » originel *pót-ih $h_{2}$, féminin de *pót-i-s « maître », qui fut ensuite modifié en *pót-n-ih $h_{2}$ sous l'influence de « reine ». On enseigne habituellement que lit. patì < * patī « maîtresse » (aussi dans viešpati) est analogique de patìs, à côté de viešpatni, féminin de viěšpats (anc. viešpatis) « seigneur ». Mais l'antiquité de *pot-ih $h_{2}$ est confirmée par tokh. B epetsa*, obl. sg. epetso « fiancée » (forme nouvelle identifiée en $\mathrm{B} 275 \mathrm{~b} 4$ ), qui présuppose un composé *n-pot-ih $h_{2}$, avec thème faible *-pot-yeh $2^{-}$, de même flexion que B lāntsa, obl. sg. lāntso « reine », qui est dérivé du nom du « roi » (thème lānt-) avec ce suffixe de féminin. Ce composé est exactement parallèle à véd. sapátnī- « co-épouse, concubine». Sur tokh. com. *pcetsā « fiancée» a été formé un masculinatif reflété par tokh. A pats « fiancé, époux », lequel ne pourrait pas refléter directement * pót-i-, qui aurait dû aboutir à tokh. com. *paecä $>\mathrm{B} * p e c, \mathrm{~A} * p a c$. Le baltique a probablement hérité des deux formations concurrentes, avec ou sans nasale, du féminin « maîtresse ». Par conséquent, la nasale insérée dans le thème *pót-n-ih $h_{2}$ 'a rien à voir avec la formation du féminin, comme on l'a parfois supposé. Pour le nom hérité de la « reine », le thème $* h_{3} r e \bar{g}-n-i h_{2}$ repose en fait sur un thème * $h_{3} r \bar{e} \hat{g}-(e) n$ - «pouvoir royal, royauté » (cf. av. rāzar-/rāzan-), et signifie « dotée de la royauté »; le doublet plus récent rájan- de ráj- « roi » repose quant à lui sur l'animation de cet abstrait. Dans le prototype *pót-ih la valeur de domination, de possession, s'est étendue de la base au suffixe. En dernière analyse, le prototype $* d i w-i h_{2}$ basé sur le thème faible de $* d y e ́ w$ - « ciel lumineux » fut restructuré en *déiw-ih 


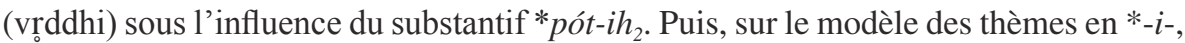
ce thème aurait adopté la flexion protérokinétique, thème faible $* d i w$-yéh ${ }_{2}$, et généralisation de l'accent sur le suffixe, d'où véd. deví-, avec en outre nivellement du vocalisme radical (cf. le nivellement inverse dans gr. $\delta \tilde{\alpha} \alpha$ ). À partir de là, la suffixation en

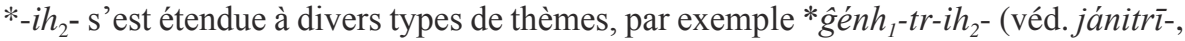
gr. $\gamma \varepsilon v \varepsilon ́ \tau \varepsilon 1 \rho \alpha$, lat. genitrīx) « maitresse de l'engendrement» (sur le nom d'action neutre * génh $h_{1}$-tro, qui fut restructuré ensuite comme féminin du nom d'agent * génh $h_{1}$-tor(véd. jánitar-, gr. $\gamma \varepsilon v \varepsilon ́ \tau \omega \rho$, lat. genitor) « géniteur ». On peut désormais mieux comprendre l'évolution du type deví- vers le procédé de formation du féminin motivé des noms athématiques, qui ne peut pas être confondu avec le type vrkíl- «louve », lequel repose sur un suffixe complexe $*_{-} i-h_{2}$ - à valeur d'appartenance, et qui n'est pas proprement féminin, cf. véd. rathí- masc. « homme en char », et le génitif thématique en $-\bar{l}$ du latin et du celtique. Les deux types en question avaient des origines totalement différentes, et il est quasi impossible de les expliquer si l'on part d'une source unique : un suffixe d'abstrait/collectif $*_{-}-i-h_{2^{-}}$; dans ce cas, pourquoi le type deví- est-il alternant, et l'autre pas? Et pourquoi le type deví- a-t-il un nominatif singulier sans désinence, à la différence de l'autre, $-\bar{l}-s$ ? Dans le type originel *pót-i-s, acc. sg. *pót-i-m, fém. * pót-ih $h_{2}$ acc. sg. *pót-ih $-m$ le morphème ajouté à la particule *pót était le pronom déictique $* i-$, sg. nom. masc. *ey, fém. ${ }^{*} i h_{2}$ (sans désinence $*_{-s} s$, qui en a fait un élément fléchi, assimilé ensuite à un nom avec suffixe $*_{-i-}:$ au masculin, le nom. sg. $*_{-} i-s$ était aligné sur l'acc. sg. *-i-m. Le nominatif sg. sans désinence du type véd. deví est l'ultime trace de cette origine pronominale. Cette démonstration ouvre des perspectives très vastes, qui ont été seulement tracées au cours de la conférence; elles seront reprises à une autre occasion. Il ne faut sans doute pas attribuer au hasard le fait que la reconstruction du processus de cristallisation d'une forme de féminin met en jeu des termes institutionnels qui reflètent la structure sociale et familiale. Nous restons donc dans la lignée de Benveniste, bien que nos résultats soient différents dans le détail.

\section{Lecture de textes tokhariens}

La partie tokharienne de l'enseignement a pris pour base un texte célèbre et méconnu à la fois, le Buddhastotra («Louange du Buddha ») de la collection Petrovskij (nom du consul russe à Kashgar, 1837-1908), de Saint-Pétersbourg. Il est contenu dans deux feuilles d'un manuscrit écrit en tokharien B, qui fut le premier document publié en Russie en 1892, en fac-similé, mais non déchiffré alors, de la langue du bassin du Tarim qui sera appelée « tokharien » après son déchiffrement en 1908. Par un paradoxe malheureusement courant dans les études tokhariennes, ce manuscrit n'avait jamais été édité en entier jusqu'à sa publication dans le chapitre XII de ma Chrestomathie tokharienne (Paris - Louvain, 2008), p. 293-311. Dans ce livre, j'ai donné une transcription du texte, sa traduction et un commentaire qui se fonde sur les strophes parallèles dans le panégyrique (stotra) de Mātrceța (actif au plus tard au IV siècle de notre ère), le Varnāarhavarṇa «Éloge de celui qui est digne d'éloge ». Cet ouvrage, dont le texte sanskrit est quasi complet, et dont il existe une traduction tibétaine, est désormais disponible dans l'édition, avec traduction allemande, de J.-U. Hartmann, Göttingen, 1987 (Sanskrittexte aus den Turfanfunden, 12). Le texte tokharien est écrit en 
tokharien B standard ou « classique » : il fournit donc une référence commode pour l'étude de la langue dans une forme normalisée, alors que beaucoup de manuscrits en tokharien B comportent des formes d'époques différentes. Il n'a pas fait l'objet d'une explication grammaticale détaillée dans ma Chrestomathie. Ce texte est suffisamment riche pour donner accès à tous les points de la morphologie et de la syntaxe tokhariennes. Les faits ont évidemment été décrits au moyen de la confrontation régulière avec l'autre langue, le tokharien A. Cette analyse a fourni aussi le prétexte pour traiter, à la demande des auditeurs, des questions d'étymologie tokharienne dans le cadre indo-européen. En particulier, on est revenu sur la question trop rapidement tranchée jusqu'à présent de l'évolution du groupe *tr en tokharien commun. Plusieurs bonnes étymologies prouvent que ce groupe évoluait en position intérieure (intervocalique) vers $*_{-} \theta r->*_{-} h r->*_{-} r$-, mais cette évolution était souvent contrariée par diverses actions analogiques; elle ne se produisait pas devant/r/ syllabique. Il arrivait que le groupe intérieur « revienne » à *-tr- à partir de la phase intermédiaire *- $\theta r$-. Par conséquent, le groupe -tr- observé dans les mots tokhariens est toujours secondaire. En début de mot, ce groupe est conservé. Une évolution du même type s'est produite en moyen-iranien, ce qui indiquerait la dimension aréale du phénomène, par ailleurs parfaitement banal sur le plan phonétique. Ce point fait l'objet d'un article en préparation. 